\title{
VARIABILIDAD EN LOS MÉTODOS DE ESTIMACIÓN DE LA CONCENTRACIÓN ESPERMÁTICA EN VERRACOS ${ }^{1}$
}

\author{
Anthony Valverde-Abarca ${ }^{2 / *}$, Mónica Madrigal-Valverde ${ }^{* * * * * *}, J_{a c o b o}$ Solís-Arias ${ }^{* * * *}$, \\ Wilfrido Paniagua-Madrigal ${ }^{* * * * * *}$
}

Palabras clave: Concentración espermática; semen; verraco; reproducción; espermatozoide.

Keywords: Sperm concentration; semen; boar; reproduction; spermatozoa.

Recibido: $11 / 06 / 18$

Aceptado: 10/01/19

RESUMEN

El análisis de la concentración espermática en los eyaculados de verraco constituye una estimación de calidad en granjas dedicadas a la venta de dosis seminales e inseminación artificial. El objetivo del presente estudio fue determinar las diferencias en precisión entre métodos de estimación de la concentración espermática. Se analizaron 174 eyaculados de verracos Duroc y Pietrain, pertenecientes a la granja del Programa de Producción Agropecuaria (PPA) de la Escuela de Agronomía del Instituto Tecnológico de Costa Rica. La concentración fue estimada por 3 métodos: espermadensímetro (ED), espectrofotómetro (EF) y sistema automatizado de análisis de semen (CASA). La concentración más baja $(\mathrm{p}<0,05)$ se

1 Trabajo formó parte del proyecto de investigación 2151-037, inscrito en la Vicerrectoría de Investigación y Extensión del Instituto Tecnológico de Costa Rica, San Carlos, Costa Rica.

2 Autor para correspondencia. Correo electrónico: anvalverde@tec.ac.cr

* Instituto Tecnológico de Costa Rica, Escuela de Agronomía, Centro de Investigación y Desarrollo en Agricultura Sostenible del Trópico Húmedo (CIDASTH), Alajuela, Costa Rica.

(iD) 0000-0002-3191-6965.

** Instituto Tecnológico de Costa Rica, Escuela de Agronomía, Centro de Investigación y Desarrollo en Agricultura Sostenible del Trópico Húmedo (CIDASTH), Alajuela, Costa Rica.

(iD) 0000-0002-4688-0627.
ABSTRACT

The variability of the estimation methods of the boar sperm concentration. The sperm concentration analysis in boar ejaculates is a quality measure in farms dedicated to the selling seminal doses and artificial insemination. The aim of this study was to determine differences in accuracy between assessment methods of sperm concentration. A total of 174 ejaculates from Duroc and Pietrain boars were analyzed. The animals belonging at the Agricultural Production Program (PPA) of the School of Agronomy at the Technological Institute of Costa Rica. The sperm concentration was estimated by 3 methods: sperm-densitometer (ED), spectrophotometer (EF) and computer assisted semen analysis (CASA).

\footnotetext{
*** Universidade Federal da Bahia, Escola de Medicina Veterinária e Zootecnia, Bahía, Brasil. (iD) 0000-0002-4688-0627.

**** Instituto Tecnológico de Costa Rica, Escuela de Agronomía, Centro de Investigación y Desarrollo en Agricultura Sostenible del Trópico Húmedo (CIDASTH), Alajuela, Costa Rica. (iD) 0000-0002-6712-1881.

****** Instituto Tecnológico de Costa Rica, Escuela de Agronomía, Centro de Investigación y Desarrollo en Agricultura Sostenible del Trópico Húmedo (CIDASTH), Alajuela, Costa Rica. (D) 0000-0002-4929-3946.
} 
estimó con el EF $(384,90 \pm 11,19$ x106 espermatozoides. $\left.\mathrm{ml}^{-1}\right)$ y la mayor con el CASA $(432,39 \pm 20,81$ $\mathrm{x} 10^{6}$ espermatozoides. $\mathrm{ml}^{-1}$ ). Se utilizó la metodología de modelos mixtos generalizados en los que incluyeron como variables explicativas el volumen de eyaculado, la edad de los verracos, la línea genética, el intervalo entre extracciones, la época y las interacciones entre los factores. El coeficiente de variación fue diferente $(\mathrm{p}<0,05)$ entre los métodos y el mayor correspondió al sistema CASA $(45,38 \%)$, mientras que el más bajo con el método ED (34,78\%). Se presentaron diferencias $(\mathrm{p}<0,05)$ entre los métodos de estimación EF y CASA $\left(376,65 \pm 12,06\right.$ vs $432,20 \pm 22,08 \times 10^{6}$ espermatozoides. $\mathrm{ml}^{-1}$ ), respectivamente, cuando el intervalo entre extracciones seminales fue bajo ( $\leq 9$ días). Sólo el sistema CASA se ajustó $(\mathrm{p}<0,05)$ al modelo y presentó un nivel asintótico de la concentración sobre el intervalo entre extracciones de 7,5 días. En general, los métodos de estimación de la concentración presentaron variabilidad en los resultados. Las líneas genéticas Duroc presentaron mayor concentración espermática que las líneas Pietrain, independientemente del método de estimación. El volumen de eyaculado presentó una correlación negativa con la concentración espermática. La concentración disminuyó conforme se incrementó el volumen de eyaculado y la edad de los verracos.

\section{INTRODUCCIÓN}

El estudio de la calidad de los eyaculados constituye un factor clave para el desarrollo y éxito de la aplicación de técnicas de inseminación artificial (IA) en especies ganaderas, los resultados de los análisis seminales tanto macro como microscópicos son de gran importancia para los programas de IA porcina (Bonet et al. 2013, Valverde et al. 2018).

Dentro de un programa de IA una de las pruebas de la evaluación seminal, que debe
The lower concentration $(\mathrm{p}<0.05)$ was estimated with EF $\left(384.90 \pm 11.19 \times 10^{6}\right.$ spermatozoa.ml $\left.{ }^{-1}\right)$ and the highest with CASA $\left(432.39 \pm 20.81 \times 10^{6}\right.$ spermatozoa $\left.\mathrm{ml}^{-1}\right)$. Concentration was analyzed using a generalized mixed model, including as explanatory variables from the model the volume of ejaculate, age of the boars, genetic line, collection interval, season and the interactions between the factors. The coefficient of variation was different $(\mathrm{p}<0.05)$ among methods and the highest was CASA system (45.38\%) while the lowest was ED method (34.78\%). There were differences $(\mathrm{p}<0.05)$ between EF and CASA estimation methods (376.65 \pm 12.06 vs $432.20 \pm 22.08 \times 10^{6}$ spermatozoa. $\mathrm{ml}^{-1}$ ) respectively, when the range between seminal extractions was low ( $\leq 9$ days). Only CASA system estimation was adjusted $(\mathrm{p}<0.05)$ and asymptotic level of concentration of 7.5 days was presented over the range between collections. In general, the estimation methods of concentration showed variability in the results. Duroc genetic lines presented higher sperm concentration than the Pietrain lines, independently of the estimation method. The volume of ejaculate presented a negative correlation with sperm concentration. The concentration decreases with increases the volume of ejaculate and the age of the boars.

considerarse, es la concentración de espermatozoides del eyaculado que es destinado para dosis seminales y cuya estimación debe ser precisa. La concentración espermática, aunque no es una estimación específica de la función testicular, se encuentra asociada a la tasa de fertilización y preñez (Vianna et al. 2004, World Health Organization 2010, Graffer et al. 1988). La estimación sesgada de la concentración espermática puede llevar a la reducción de la fertilidad de las hembras, lo cual aumenta el retorno al estro y 
disminuye tasas de parto, tamaño de camada y aumento en el costo por dosis. La sobre estimación de la concentración espermática significa que la estimación es mayor que la concentración real en el eyaculado (Krueger et al. 1999, Vianna et al. 2004) y como consecuencia, se añade una cantidad inadecuada de diluyente que altera el número de dosis por eyaculado y por otra parte, la subestimación conduce a pérdidas económicas en la granja. Esos aspectos implican que, si no es estimada adecuadamente, la concentración de espermatozoides se puede generar un número de dosis seminales no adecuado, con menor o mayor número de células por dosis, lo que afecta al comprador o vendedor de dosis seminales, respectivamente.

El método utilizado para la determinación de concentración espermática es un factor relevante a considerar para una adecuada estimación. Los métodos empíricos de determinación de la concentración, basados en la evaluación por el aspecto físico del eyaculado, son extremadamente imprecisos y subjetivos, por lo que es requerido el empleo de métodos directos de determinación con mayor confiabilidad (Bortolozzo et al. 2005). Estos métodos subjetivos requieren el uso de cámaras en las que las células se observan y contabilizan dentro de un área conocida, lo cual permite luego, el cálculo del número de espermatozoides por unidad de volumen (Brito et al. 2016).

El espermadensímetro es uno de los métodos visuales que se utilizan para la estimación de concentración espermática y esta herramienta ofrece una estimación rápida con base en el grado de turbidez o densidad del eyaculado. La lectura del instrumento presenta un grado de subjetividad, además, de la propia densidad del eyaculado, que puede ser influenciada por el número de leucocitos, células de descamación o partículas de suciedad provenientes del eyaculado (Vianna et al. 2004).

El espectrofotómetro se ha desarrollado como alternativa a los hemacitómetros y consiste en una técnica de lectura de la concentración realizada a través del grado de absorbancia de la luz en el eyaculado. Este método ofrece rapidez y evaluaciones precisas cuando el equipo se encuentra calibrado; no obstante, presenta algunas deficiencias, tales como que las concentraciones espermáticas solo pueden ser leídas dentro de cierta franja y que las lecturas se ven afectadas por células de descamación, leucocitos y suciedad (Brito et al. 2016, Vianna et al. 2004).

Los sistemas automáticos de análisis de semen (Computer assisted semen analysis, CASA) son cada vez más comunes en los centros de inseminación artificial y granjas de verracos (Valverde y Madrigal-Valverde 2018); poseen un sistema óptico con iluminación por contraste de fase, una cámara digital que graba a un número determinado de imágenes por segundo; por lo general, se utiliza una frecuencia de $50 \mathrm{fps}$ ( $\mathrm{fra}$ mes per second) durante 1-2 segundos, un sistema computacional y un monitor de video (Amann y Waberski 2014, Vásquez et al. 2016, Valverde et al. 2018). Este sistema permite analizar la calidad del movimiento espermático a través del análisis de imagen y como consecuencia, de que el área de las imágenes es conocida y el volumen evaluado, se puede estimar la concentración espermática. Además, los sistemas CASA aportan nuevos parámetros avanzados de cinética celular y morfología (Mortimer 2000).

Desde el punto de vista de comercialización de dosis seminales, la cantidad y la calidad del semen son los 2 factores principales que afectan el número de dosis que se pueden producir por eyaculado (Flowers 1997) por lo que la variabilidad espermática que surge de los factores exógenos y endógenos (Ciereszko et al. 2000) y las características seminales como volumen, concentración, movilidad y morfología presentan variabilidad inter e intra-razas, deben ser identificadas para evaluar la calidad del semen (Smital 2009). El objetivo del presente estudio fue determinar las diferencias en precisión existentes entre los métodos de estimación espermática de la forma más aproximada y objetiva posible. 


\section{MATERIALES Y MÉTODOS}

\section{Descripción y localización de la granja}

El presente estudio se realizó en la granja del Programa de Producción Agropecuaria (PPA), de la Escuela de Agronomía, del Instituto Tecnológico de Costa Rica, ubicado en Santa Clara, Florencia, San Carlos, Alajuela, Costa Rica $\left(10^{\circ} 22^{\prime} 42^{\prime \prime} \mathrm{N}\right.$ y 8430’36” O), durante julio de 2014 hasta febrero de 2016. La unidad productiva se encuentra a una altitud de $120 \mathrm{msnm}$, según los datos registrados en la Estación Meteorológica 069567 Sta. Clara. En el área donde se encuentra la porqueriza, se registró durante el quinquenio 2010-2015, una temperatura mínima y máxima de $21,7^{\circ} \mathrm{C}$ y $30,7^{\circ} \mathrm{C}$, respectivamente, una humedad relativa de $88,5 \%$, con un régimen de lluvias de $3321,1 \mathrm{~mm}$ al año en promedio. La granja tiene como objetivo comercial la venta de lechones al destete (30 días de edad). Se mantiene una piara de 20 hembras y el reemplazo se compra a una casa comercial.

\section{Animales}

\section{Manejo y descripción de los animales}

En la granja los machos se mantuvieron en recintos individuales de un mínimo de 7,5 $\mathrm{m}^{2}$ y la alimentación de los animales fue con base en un alimento balanceado, formulado por la misma universidad y ofrecido a razón de 2,5 kg.animal ${ }^{-1} \cdot$ día $^{-1}$. El agua fue suministrada ad libitum. Se utilizaron 4 verracos, 2 verracos de cada línea genética estudiada en buen estado general de salud y en edad reproductiva con edades superiores a los 15 meses. La base racial de la línea genética A fue Pietrain, mientras que para la línea genética $B$ fue Duroc. La frecuencia de extracción fue 2 veces cada semana. El intervalo entre extracciones seminales (I) fue categorizado en 2 grupos: I-Alto: $>9$ días, I-Bajo: $\leq 9$ días. Asimismo, el volumen (vol) eyaculado jerarquizó en 2 grupos: volumen eyaculado alto: $>250 \mathrm{ml}$; volumen eyaculado bajo: $\leq 250 \mathrm{ml}$. Los animales se emplearon en cruzamientos terminales, con el fin de aportar aptitudes cárnicas a la progenie y procedían de dos líneas genéticas comerciales seleccionadas para producción de carne magra y ganancia de peso. Los animales fueron entrenados para colección de semen en un "maniquí", o potro de monta, e incorporados a un programa de inseminación artificial. Los animales no fueron empleados como reproductores en la granja en periodos simultáneos.

\section{Recolección de muestras de semen y procesamiento}

Se recolectaron muestras de semen fresco y se diluyeron 1:1 (vol:vol) después de cada extracción; se utilizó la técnica de la "mano enguantada"(Hancock y Hovell 1959) para obtener semen para inseminación artificial. Antes de cada eyaculación, se estimulaba a los verracos al llevarlos a un corral de extracción separado, que contenía el potro de extracción o "maniquí" y se obtenía el semen mediante manipulación manual del pene después de que el verraco montara el potro de extracción. Las 3 últimas fracciones de semen de cada eyaculado se recogieron en recipientes graduados de recogida de esperma y se filtraron a través de 3 capas de gasa esterilizada para separar las secreciones de glándula bulbouretral de los otros constituyentes del semen. Después de cada recolección de semen, se realizó una evaluación macroscópica de rutina (volumen, color, consistencia y olor). Después de esta evaluación, que tardó varios segundos, se colocó la fracción filtrada en un baño maría a $37^{\circ} \mathrm{C}$, donde las muestras permanecieron durante el periodo de envasado y determinación de la densidad del semen.

\section{Medición de la concentración espermática}

Para la medición de la concentración espermática con el espectrofotómetro, se tomó una alícuota de semen diluido 1:1 (vol:vol) menor a $1 \mathrm{ml}$ por medio de una pipeta Pasteur y se colocó en una micro cubeta antes de realizar 
la medición. Dicha estimación fue tomada por triplicado con la finalidad de estimar un promedio. Para la determinación de la concentración con el espermadensímetro de Karras, se tomó del mismo eyaculado con una pipeta graduada, una parte de semen y nueve partes de medio de dilución (diluyente comercial BTS + agua destilada a una temperada de $37^{\circ} \mathrm{C}$ ) y se depositaron en un beaker de $50 \mathrm{ml}$. El contenido fue homogenizado $\mathrm{y}$, posteriormente, se colocó en el instrumento de medición. Se realizó la lectura del aparato siguiendo las indicaciones del fabricante y se registró la estimación por triplicado junto a las anteriores evaluaciones. Para la evaluación de la concentración, se utilizó un sistema CASA comercial y se utilizaron cámaras de recuento de $20 \mu \mathrm{m}$ de altura. Se agregaron $2,7 \mu \mathrm{L}$ de semen a cada cámara de recuento y se dejó avanzar por capilaridad a través de la misma. Los espermatozoides se contabilizaron mediante microscopía de contraste de fase negativo a 40X aumentos. El número de campos en cada análisis fue determinado según el número de células y en promedio se capturaron 7 campos. Los recuentos del sistema CASA se realizaron corregidos, es decir, se obtuvieron al repetir continuamente un video de un segundo campo tomado aleatoriamente de la cámara D4C20. Cada campo fue seleccionado manualmente por el técnico. Todos los espermatozoides visibles en ese campo se contabilizan manualmente y los resultados se dividieron por un factor de 6 que se convierte en $\times 10^{6} \cdot \mathrm{ml}^{-1}$. El recuento se realizó de acuerdo con las directrices de la OMS para recuentos manuales (World Health Organization 2010). Posteriormente a la evaluación de concentración espermática, el eyaculado fue procesado para elaborar dosis seminales.

\section{Análisis estadístico}

Se realizaron pruebas de normalidad y homocedasticidad usando Shapiro-Wilk y pruebas de Kolmogorov-Smirnov, respectivamente, a los datos obtenidos de las estimaciones de concentración espermática. La repetibilidad de cada método de medición se evaluó mediante el coeficiente de variación (CV) y se calculó al estimar la variación usando un análisis de comparación por pares separado para cada método. El eyaculado se incluyó como efecto aleatorio en la estructura del modelo. La comparación de métodos se realizó utilizando la transformación logarítmica de las mediciones de concentración de espermatozoides. Para la comparación de métodos se utilizó un modelo mixto. En el análisis, la varianza para cada método se estimó por separado.

El modelo estadístico utilizado fue del tipo:

$$
Y_{i j k l m n o}=\mu+M_{i}+E_{j}+A_{k}+I_{L}+E P_{M}+R_{n}+(M I)_{i l}+(M R)_{i n}+\varepsilon_{(i j k l m n) o}
$$

Donde,

$\boldsymbol{Y}_{\text {ijklmno }}=$ o-ésimaobservación correspondiente ali-ésimofactor $\mathrm{M}$, del $j$-ésimofactor $\mathrm{E}$, en el $k$-ésimo factor $\mathrm{A}$, del $l$-ésimo del factor I en el nivel $m$-ésimo del factor EP dentro del nivel $n$-ésimo del factor R (observación $=1,2, \ldots, o$ ). $\mu=$ media general.

$\boldsymbol{M}_{\boldsymbol{i}}=$ efecto del nivel $i$-ésimo del factor M (método; $i=1,2,3)$.

$\boldsymbol{E}_{j}=$ efecto del nivel j-ésimo del factor $E$ (eyaculado; $j=1,2,3)$.
$\boldsymbol{A}_{\boldsymbol{k}}=$ efecto del nivel $k$-ésimo del factor A (año; $k=1,2,3)$.

$\boldsymbol{I}_{\boldsymbol{L}}=$ efecto del nivel $l$-ésimo del factor I (intervalo entre extracciones; $l=1,2, \ldots, i)$.

$\boldsymbol{E P}_{\boldsymbol{M}}=$ efecto del nivel m-ésimo del factor EP (época; $\mathrm{m}=1,2$ ).

$\boldsymbol{R}_{N}=$ efecto del nivel n-ésimo del factor $\mathrm{R}$ (grupo racial; $\mathrm{n}=1,2,3)$.

$(M I)_{i l}=$ efecto producido por la interacción $\mathrm{M} * \mathrm{I}$. $(\boldsymbol{M R})_{i n}=$ efecto producido por la interacción $\mathrm{M} * \mathrm{R}$.

$\varepsilon_{(i j k l m n) o}=$ error experimental. Se suponen variables aleatorias independientes $N(0, \sigma)$. 
El efecto de la edad de los verracos, el volumen de eyaculado y el intervalo entre extracciones seminales sobre la concentración espermática, se analizó mediante regresión no lineal con un modelo exponencial, en la forma $\mathrm{y}=\beta$ $* \alpha \exp (-\beta / \mathrm{x})$, donde $y$ era concentración seminal $\left(\mathrm{x} 10^{6}\right.$ espermatozoides. $\mathrm{ml}^{-1}$ ) y $x$ fue la edad del verraco; el intervalo entre extracciones y el volumen de eyaculado de forma independiente donde $a=$ nivel asintótico, $b=$ tasa de aumento a la asíntota, exp = base del logaritmo natural. El significado biológico de la ecuación es que los valores asintóticos (a) representan el máximo alcanzable cuando la edad está por encima del nivel del umbral. El nivel de umbral se calculó por el método convencional que evalúa la edad, el intervalo o el volumen necesario para obtener el 95\% del valor máximo. La tasa de acercamiento al nivel asintótico representa la dependencia de la curva en la edad de los animales; es decir, un valor alto de $b$ indica un alto crecimiento de la concentración a medida que el intervalo entre extracciones seminales aumenta y viceversa. Un valor bajo de $b$ indicará una disminución en la concentración a medida que la edad de los animales y el volumen de eyaculado aumenten y viceversa.

Se realizaron pruebas de comparación múltiple por el método de mínimos cuadrados y se utilizó la corrección de Bonferroni, con un nivel de significación estadística de 0,05. Los análisis estadísticos se realizaron con el programa StatgraphicsCenturion XVII, 17.2.04. (32-bit) (1982-2016 por Statpoint Technologies, Inc., EE. UU.).

\section{RESULTADOS}

\section{Estimación de la concentración espermática}

Los métodos de estimación de la concentración de espermatozoides presentaron diferencias $(p<0,05)$ entre sí. El equipo que estimó la concentración espermática más alta fue el CASA, mientras que la menor fue con el espectrofotómetro, con una diferencia entre métodos de $4,75 \times 10^{7}$ espermatozoides por $\mathrm{ml}$. El coeficiente de variación fue estadísticamente distinto entre los diferentes métodos utilizados y el mayor coeficiente fue presentado por el método de estimación CASA (Cuadro 1).

Cuadro 1. Medias ( \pm E.E.) y resultados descriptivos de los métodos de medición de la concentración espermática en verracos $(n=174)$

\begin{tabular}{|c|c|c|c|}
\hline & Espermadensímetro & Espectrofotómetro & CASA \\
\hline Concentración $\left(\times 10^{6} \cdot \mathrm{ml}^{-1}\right)$ & $405,98 \pm 11,19^{\mathrm{ab}}$ & $384,90 \pm 11,19^{\mathrm{a}}$ & $432,39 \pm 20,81^{\mathrm{b}}$ \\
\hline D.E. & 141,21 & 153,76 & 196,21 \\
\hline $\mathrm{CV}(\%)$ & $34,78^{\mathrm{a}}$ & $39,95^{\mathrm{b}}$ & $45,38^{\mathrm{c}}$ \\
\hline $\mathrm{Li}(95 \%)$ & 390,43 & 369,35 & 403,46 \\
\hline Ls $(95 \%)$ & 421,53 & 400,45 & 461,31 \\
\hline Media dosisseminales (n) ${ }^{*}$ & 24,9 & 23,6 & 28,8 \\
\hline
\end{tabular}

E.E.: error estándar; CASA: sistema automático de análisis de semen; D.E.: desviación estándar; CV: coeficiente de variación; Li: Intervalo de confianza inferior al 95\% de probabilidad; Ls: Li: Intervalo de confianza superior al 95\% de probabilidad. ${ }^{*}$ Concentración espermática x volumen / 4000 (no considera movilidad y las dosis son fijadas a 4x109 espermatozoides totales. $\mathrm{a}, \mathrm{b}, \mathrm{c}$ Dentro de fila, valores con diferente superíndice presentan diferencias significativas. $\mathrm{p}<0,05$. 
La mayor variabilidad en la estimación de la concentración espermática se presentó en los métodos de medición con el espectrofotómetro y el sistema CASA. No hubo valores extremos ni puntos influyentes en ninguno de los 3 métodos de estimación (Figura 1).

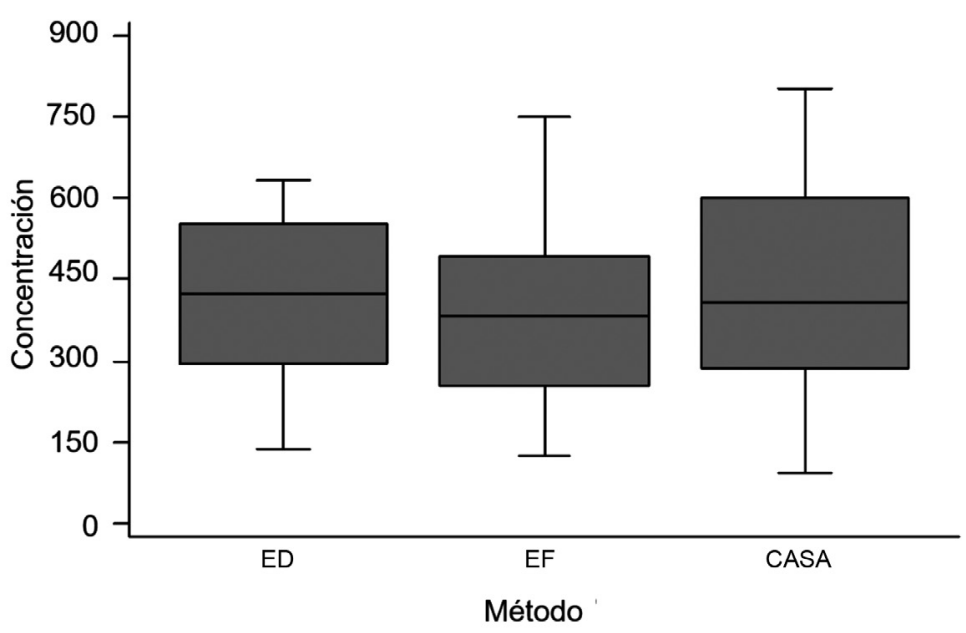

Fig. 1. Concentración espermática $\left(\times 10^{6} \cdot \mathrm{ml}^{-1}\right)$ según método de estimación. ED: espermadensímetro EF: Espectrofotómetro; CASA: sistema automático de análisis de semen. Área dentro del boxplot, indica el 50\% de las observaciones entre el

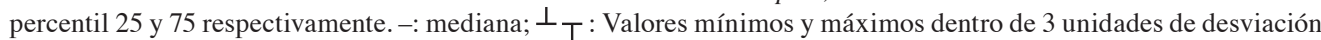
estándar.

El efecto del intervalo entre extracciones seminales influyó en la estimación de la concentración espermática. Los animales sometidos a un ritmo de extracciones elevado ( $\leq 9$ días) presentaron diferencias $(\mathrm{p}<0,05)$ entre métodos de estimación de la concentración, particularmente los métodos de medición por espectrofotómetro y
CASA. Cuando el intervalo entre extracciones seminales fue alto ( $>9$ días), es decir, un ritmo menor de extracciones de semen, no hubo diferencias entre los métodos de estimación de la concentración. Dentro de método no hubo diferencias en las estimaciones de la concentración entre los intervalos entre extracciones alto y bajo (Cuadro 2). 
Cuadro 2. Efecto del intervalo entre extracciones seminales (I), grupo racial, volumen eyaculado y época (medias \pm E.E.) sobre el método de medición de la concentración espermática.

\begin{tabular}{|c|c|c|c|}
\hline & Espermadensímetro & Espectrofotómetro & CASA \\
\hline \multicolumn{4}{|c|}{ Intervalo (I) } \\
\hline Alto & $419,36 \pm 30,04^{\text {ay }}$ & $428,68 \pm 30,15^{\text {ay }}$ & $410,05 \pm 61,15^{\text {ay }}$ \\
\hline Bajo & $403,85 \pm 12,16^{\text {aby }}$ & $376,65 \pm 12,06^{\text {by }}$ & $432,20 \pm 22,08^{\text {ay }}$ \\
\hline \multicolumn{4}{|c|}{ Grupo Racial } \\
\hline Pietrain & $417,54 \pm 11,96^{\text {ay }}$ & $394,42 \pm 11,52^{\text {ay }}$ & $469,58 \pm 21,11^{\text {by }}$ \\
\hline Duroc & $551,67 \pm 23,47^{\mathrm{az}}$ & $536,85 \pm 23,47^{\mathrm{az}}$ & $588,49 \pm 41,54^{\mathrm{bz}}$ \\
\hline \multicolumn{4}{|c|}{ Volumen eyaculado } \\
\hline Alto & $330,98 \pm 16,10^{\text {ay }}$ & $304,80 \pm 15,90^{\text {ay }}$ & $286,37 \pm 31,92^{\text {ay }}$ \\
\hline Bajo & $442,03 \pm 13,08^{\mathrm{az}}$ & $423,52 \pm 12,89^{\mathrm{az}}$ & $506,61 \pm 23,85^{\text {bz }}$ \\
\hline \multicolumn{4}{|l|}{ Época } \\
\hline Seca & $421,90 \pm 15,09^{\text {ay }}$ & $412,92 \pm 15,01^{\text {ay }}$ & $441,76 \pm 21,73^{\text {ay }}$ \\
\hline Lluviosa & $394,77 \pm 13,94^{\mathrm{abz}}$ & $365,27 \pm 13,54^{\mathrm{bz}}$ & $386,27 \pm 44,94^{\mathrm{az}}$ \\
\hline
\end{tabular}

E.E.: error estándar; CASA: sistema automático de análisis de semen; I: Intervalo entre extracciones. I-Alto: $>9$ días, I-Bajo: $\leq 9$ días; volumen eyaculado alto: $>250 \mathrm{~mL}$; volumen eyaculado bajo: $\leq 250 \mathrm{~mL}$. ${ }^{\mathrm{a}, \mathrm{b}}$ Dentro de fila, valores con diferente superíndice presentan diferencias significativas. ${ }^{\mathrm{x}, \mathrm{y}, \mathrm{z}}$ Dentro de columna, valores con diferente superíndice presentan diferencias significativas $\mathrm{p}<0,05$.

Las estimaciones de la concentración de acuerdo con el grupo racial, fueron mayores con el sistema CASA, seguidas del espermadensímetro y el espectrofotómetro, no se detectaron diferencias ( $>00,05)$ entre estos últimos. Dentro de método, se encontraron diferencias entre grupos raciales, al presentar la raza Duroc mayores concentraciones espermáticas $(p<0,05)$. Dentro de raza las estimaciones realizadas con el sistema CASA fueron mayores $(\mathrm{p}<0,05)$, que las realizadas con el espermadensímetro y espectrofotómetro, para ambas líneas genéticas. Cuando el volumen de eyaculado fue alto, no presentaron diferencias estadísticas entre los métodos de estimación, sin embargo, para volúmenes bajos de eyaculado $(\leq 250 \mathrm{ml})$ el método CASA estima la concentración más alta con respecto a los métodos ED y EF, y presenta diferencias $(\mathrm{p}<0,05)$ con respecto a ambos métodos.
El contenido espermático estimado en época seca fue mayor $(\mathrm{p}<0,05)$ que el estimado en época lluviosa para las 3 formas de medición. Dentro de época, la concentración estimada en época seca no fue diferente entre los métodos de estimación, mientras que en época lluviosa, el espectrofotómetro fue el procedimiento que generó estimaciones más bajas de presencia espermática y fue diferente $(\mathrm{p}<0,05)$ de la concentración estimada con los métodos ED y CASA (Cuadro 2).

El análisis de correlación lineal entre los métodos de estimación de la concentración, fue fuerte y positivo $(0,96)$ entre los métodos ED y EF. La correlación entre los procedimientos ED y CASA fue la menor con un valor de 0,23 y entre $\mathrm{EF}$ y CASA lineal fue positiva, pero de baja magnitud $(0,30$; Figura 2). 


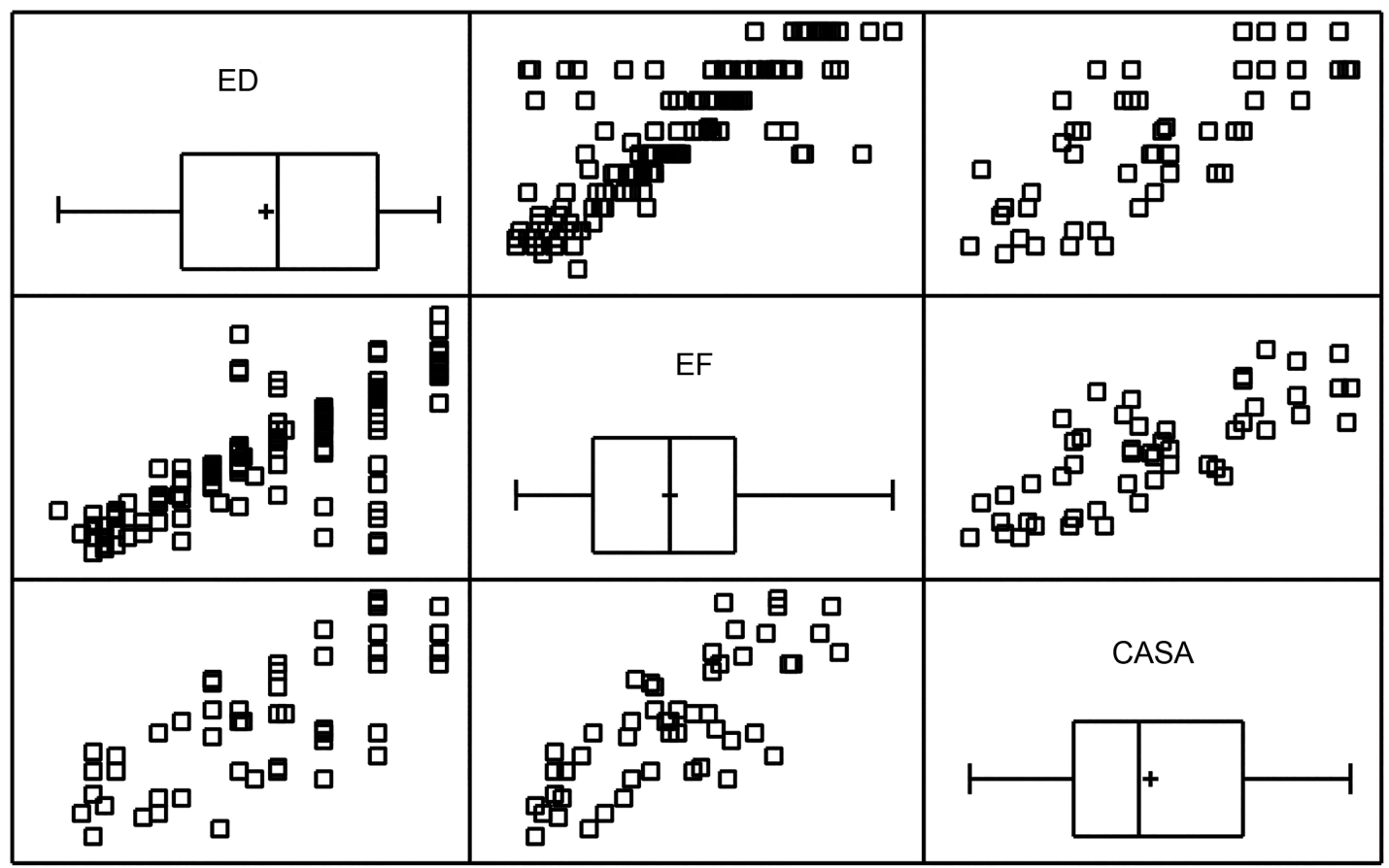

Fig. 2. Correlaciones entre los sistemas de medición de la concentración espermática. ED: espermadensímetro; EF: espectrofotómetro; CASA: sistema computadorizado de análisis de semen.

La interacción entre el método de estimación de la concentración y el intervalo entre extracciones fue significativa $(\mathrm{p}<0,05)$ y mostró que cuando el intervalo entre extracciones seminales es alto, los 3 métodos proporcionaron contenidos con mayor similitud. Por el contrario, cuando los verracos son sometidos a un ritmo mayor de extracciones seminales, los métodos resultaron más inexactos al estimar la densidad de semen en el eyaculado (Figura 3A). El sistema CASA tiende a estimar mayores aglutinaciones de semen, cuando los verracos son sometidos a un ritmo de extracciones menores, mientras que cuando los animales se someten a ritmos de extracción mayor, el CASA tiende a estimar menor la concentración espermática con respecto a los métodos EF y ED. 
A

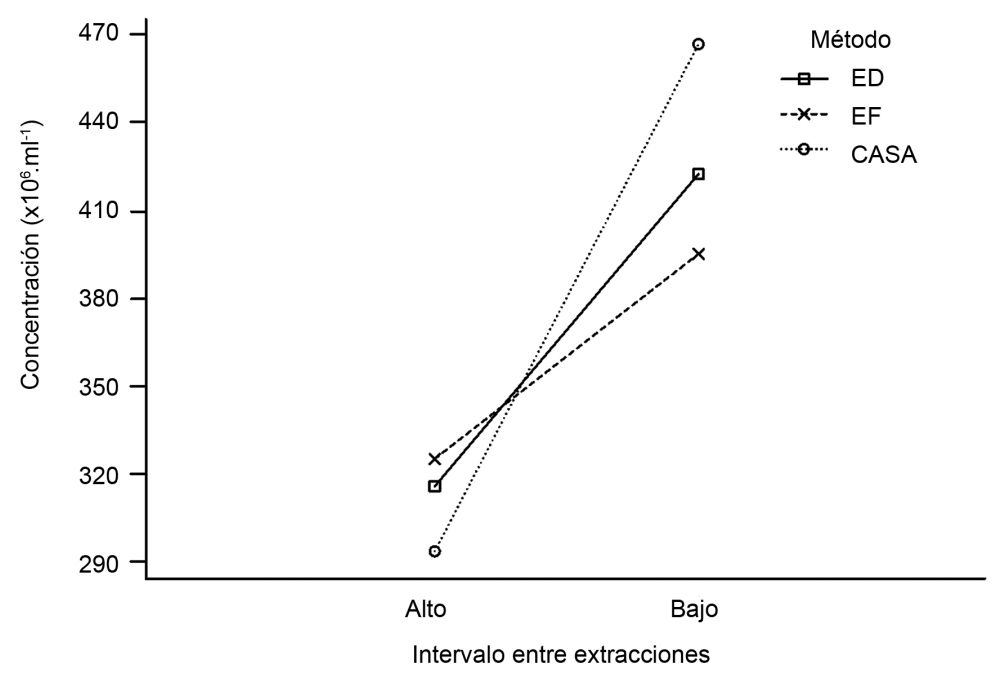

B

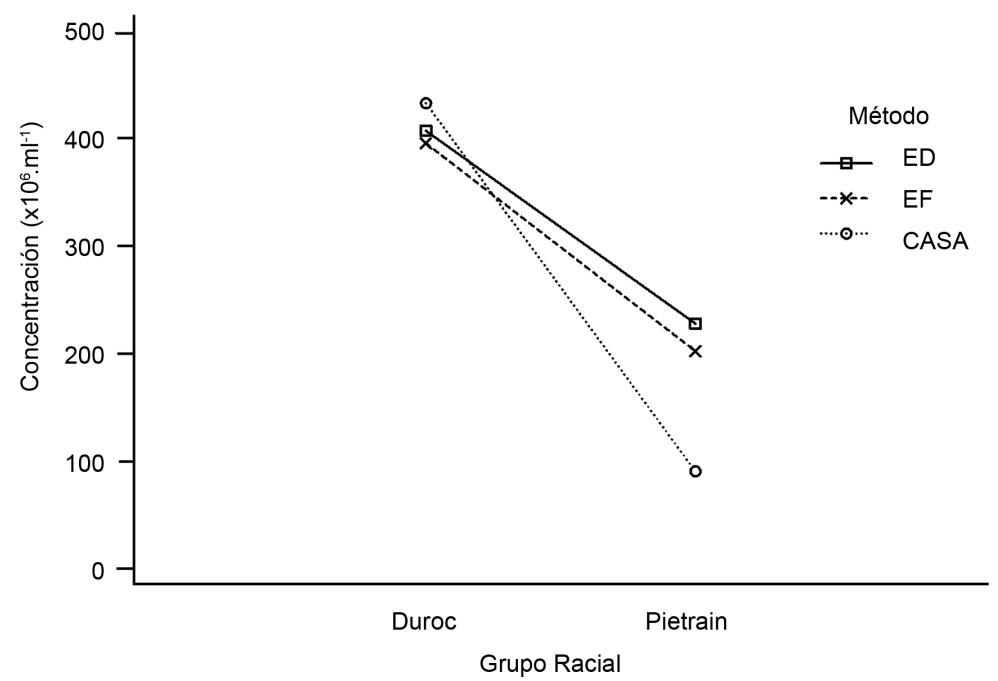

Fig. 3. A. Interacción entre método de estimación de la concentración e intervalo entre extracciones. $B$. Interacción entre método de estimación de la concentración y grupo racial.

Los 3 métodos de estimación de la concentración presentaron valores similares cuando el grupo racial fue Duroc, sin embargo, en los animales Pietrain las estimaciones con el ED y EF fueron similares, pero la estimación con el sistema CASA fue significativamente menor (Figura 3B).
El efecto del intervalo entre extracciones sobre la concentración espermática, calculada con los 3 métodos de estimación, se estudió al aplicar un modelo de regresión no lineal. El espermadensímetro y el espectrofotómetro no se ajustaron $(\mathrm{p}>0,05)$ al modelo. La estimación de la concentración por el 
sistema CASA mostró un efecto significativo $(p<0,05)$ sobre el intervalo entre extracciones y, el nivel asintótico, se estimó a partir de 7,5 días (Figura 4).

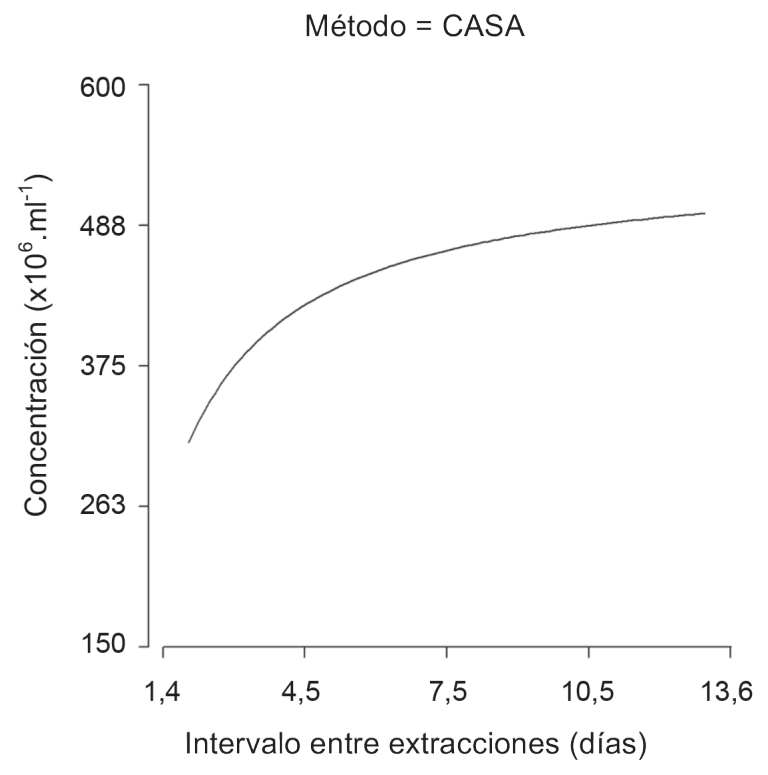

Fig. 4. Efecto del intervalo entre extracciones seminales sobre la concentración espermática estimada con un sistema CASA mediante un modelo de regresión no lineal. Modelo: $y=\beta \times \alpha \exp (-\beta / x)$.

No hubo efecto cuadrático significativo en la superficie de respuesta estimada, pero sí hubo un efecto lineal $(p<0,05)$. Los valores más altos de concentración se encuentran en la esquina inferior derecha de la gráfica de superficie de respuesta, que coincide con los valores menores de volumen de eyaculado y mayores intervalos entre extracciones. Los valores más bajos de concentración espermática se encuentran en la esquina superior izquierda, que coincide con valores altos de volumen eyaculado y menores intervalos entre extracciones seminales (Figura 5). 


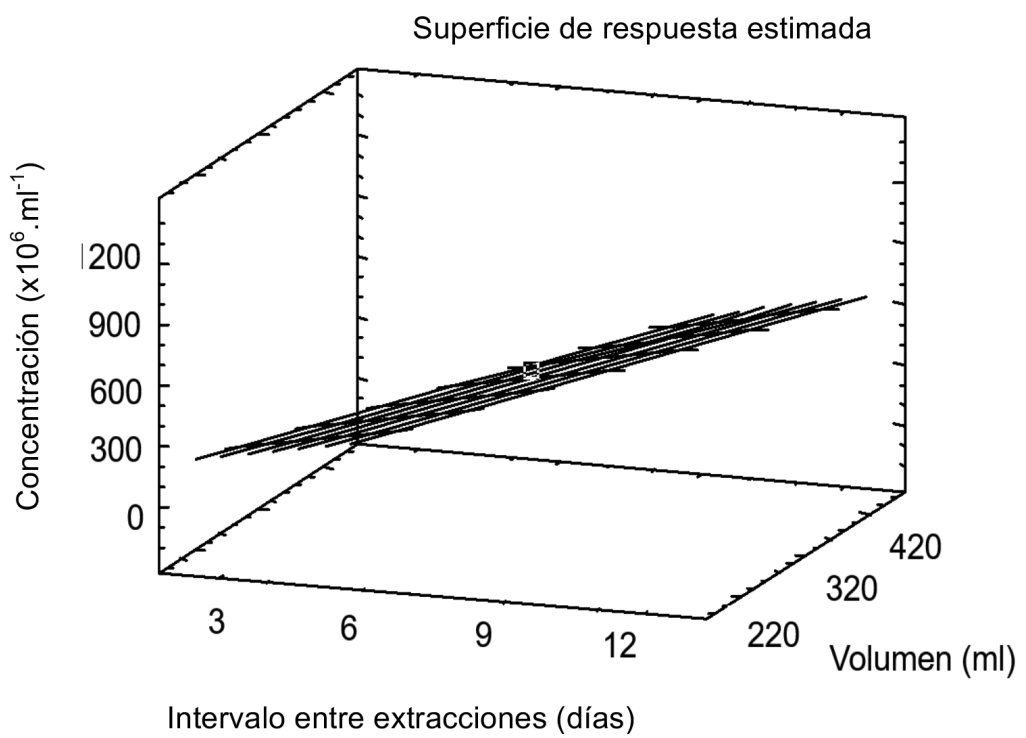

Fig. 5. Gráfica de superficie de respuesta de concentración espermática vs intervalo entre extracciones seminales vs volumen. Valores fijos: $\mathrm{Edad}=36$ meses.

El efecto de la edad de los animales, sobre la estimación de la concentración espermática, mostró para todos los métodos de estimación una reducción de la concentración conforme se incrementa la edad de los verracos. Esta reducción fue más marcada en las estimaciones realizadas con el espermadensímetro y menores con el sistema CASA (Figura 6). El análisis de regresión no lineal, entre volumen eyaculado y concentración, presentó una disminución en los valores de concentración de semen, conforme el volumen de eyaculado se incrementaba. Esta disminución fue más pronunciada en volúmenes inferiores a $250 \mathrm{ml}$, para las estimaciones de la concentración realizadas con el espermadensímetro y espectrofotómetro que con en el método CASA (Figura 7). 

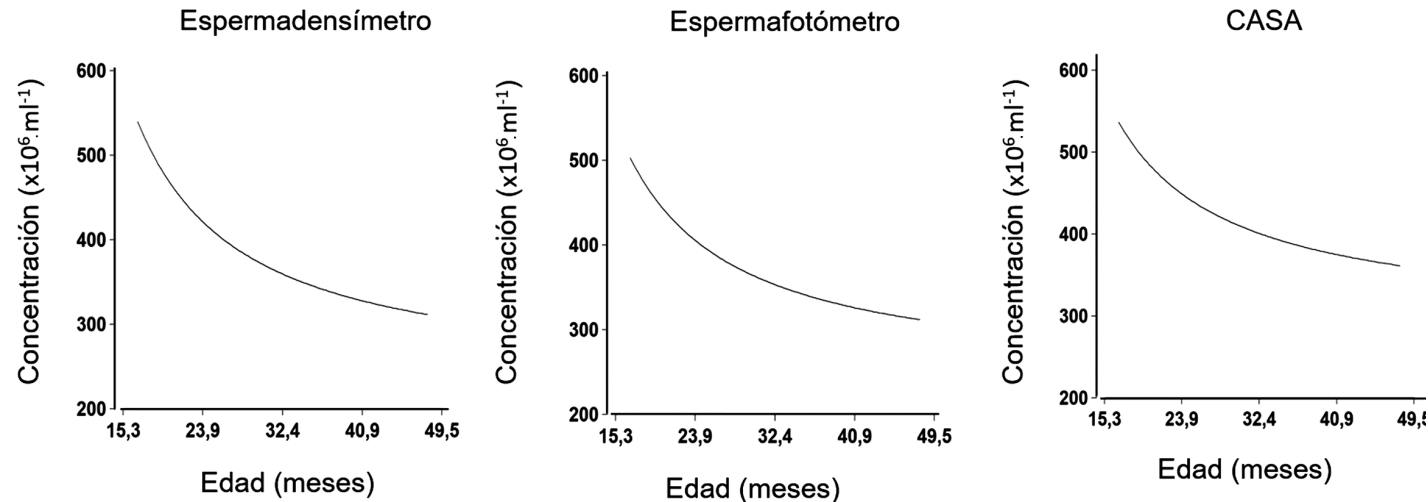

Fig. 6. Análisis de regresión no lineal. Efecto de la edad sobre la concentración espermática con 3 métodos de medición.
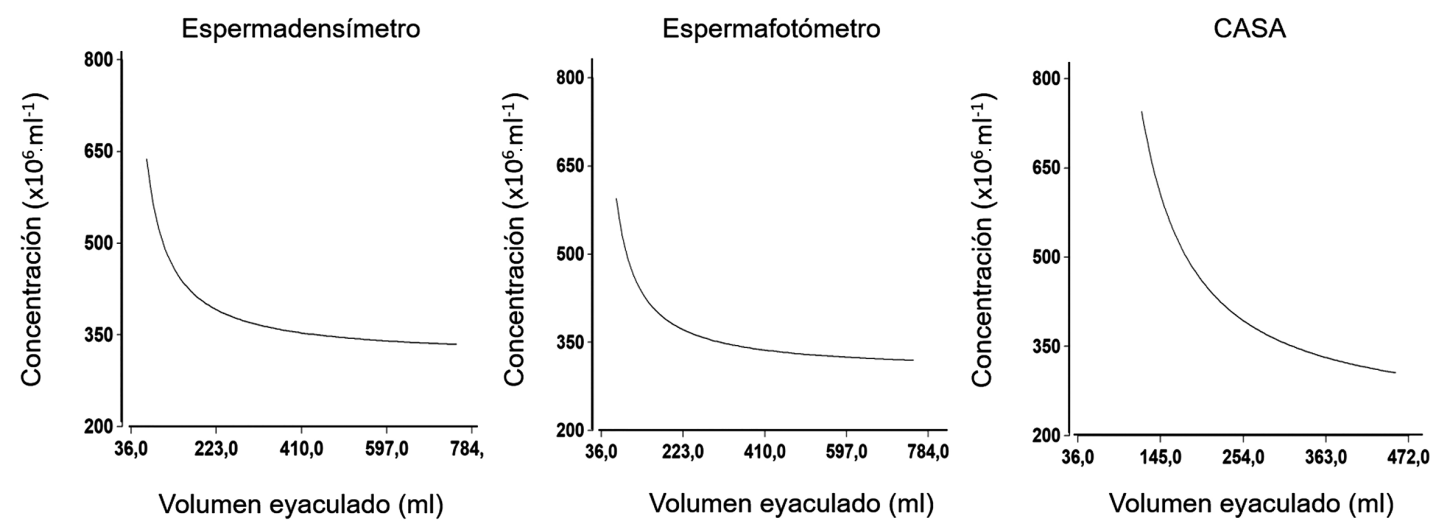

Fig. 7. Análisis de regresión no lineal. Efecto del volumen sobre la concentración espermática con 3 métodos de medición.

\section{DISCUSIÓN}

Se han reportado coeficientes de variación entre laboratorios, para la estimación de la concentración espermática con valores que van desde $23 \%$ hasta $80 \%$ en humano (Neuwinger et al. 1990, Keel 2002) y de 11 hasta $26 \%$ en porcino (Ehlers et al. 2011). Aunque se han informado pocos estudios similares en la literatura veterinaria, la situación de la andrología animal en los laboratorios, probablemente no sea muy diferente (Brito et al. 2016). Resultados con un amplio margen de variabilidad (rango $23 \%$ a $73 \%$ ) fueron reportados por Neuwinger et al. (1990) en una muestra de 10 laboratorios alemanes con experiencia en la evaluación espermática, mientras que Matson (1995) reportó coeficientes de variación inter-individual, para concentración de espermatozoides a partir de los datos del control 
de calidad externo, obtenidos en la British Fertility Society del 64,7\% en 24 muestras de semen recogidas por técnicos de 20 laboratorios. En el presente trabajo el coeficiente de variación más elevado correspondió al método CASA, con un valor de 45,38\%. El alto coeficiente de variación mostrado, por los resultados con el sistema CASA, se podría explicar también, porque en algunas ocasiones el software de análisis no reconoce o excluye partículas no espermáticas; espermatozoides estáticos muy pequeños y poco brillantes no son reconocidos por el sistema, aglutinaciones de espermatozoides o inapropiadas diluciones de la muestra, para lograr estimar con precisión la concentración óptima (Tomlinson et al. 2010). A pesar de que los coeficientes de variación encontrados en el presente trabajo están por encima del rango comparable con otros trabajos en la misma especie, por lo general, solo un tercio o menos de los casos realmente se encuentran dentro del $10 \%$ de variación (Mortimer et al. 1995). La precisión de las estimaciones de concentración obtenidas con el uso de sistemas CASA puede verse afectada, porque cada sistema automatizado tiene su propio hardware y software; además, se deben tener consideraciones sobre el tipo de cámara y la preparación de la muestra que podrían introducir variación en las estimaciones.

La concentración espermática presenta variación en los machos de diferentes especies. Está condicionado tanto por factores genéticos como ambientales que afectan la calidad y cantidad del eyaculado (Holt et al. 1997). Algunos estudios han revelado variabilidad en los parámetros espermáticos morfométricos en relación con la concentración del semen en eyaculados de perros (Rijsselaere et al. 2004), equinos (Davis et al. 1993) y verracos (Banaszewska et al. 2009, Kondracki et al. 2011). Otras investigaciones indican diferencias en las dimensiones y formas de los espermatozoides dentro de una misma especie, también entre razas de una misma especie como en verracos (Kondracki et al. 2005, Saravia et al. 2007).

La concentración estimada con el sistema CASA fue mayor que la estimada con el espectrofotómetro. La estimación realizada con el espermadensímetro no presentó diferencias estadísticas con los 2 métodos de estimación anteriores. $\mathrm{Al}$ respecto, las mediciones realizadas con el espermadensímetro están sujetas a fuentes de variabilidad externas, como partículas de suciedad, espermatozoides inmaduros, células sanguíneas y epiteliales, número de leucocitos y residuos celulares, que aumentarían la turbidez del semen y podría tenderse a sobrestimar los resultados (Eustache et al. 2001). La estimación de la concentración con el espectrofotómetro fue la menor, sin embargo, mostró un coeficiente de variación del orden del $40 \%$, que fue mayor que el reportado en otros trabajos con el mismo método (Zrimšek 2011). La curva de calibración de este instrumento puede influir en las mediciones y subestimar los resultados. El uso de controles de semen en muestras con presencia de partículas, junto con el uso de diferentes líneas de calibración en función del control, se ha descrito como una alternativa para aumentar la precisión y exactitud de los resultados del espectrofotómetro (Emik y Sidwell 1947).

Los resultados observados en este trabajo para la estimación de la concentración con los métodos ED y EF fueron mayores que los reportados por (Vianna et al. 2004) en semen de verraco, sin embargo, los coeficientes de variación fueron menores al indicar que las estimaciones en el presente trabajo fueron más precisas. Por otra parte los valores estimados de concentración, con estos 2 métodos fueron inferiores a los reportados por Murgas et al. (2010). Diferencias entre métodos de medición por espectrofotómetro y sistemas CASA han sido reportados previamente (Eljarah et al. 2013). En relación con la exactitud de los datos, los resultados muestran una menor variabilidad cuando se utilizó el espermadensímetro, lo que es contrario a Murgas et al. (2010), quienes encontraron una menor variabilidad al utilizar el espectrofotómetro en verracos. Datos muy inferiores a los encontrados en este trabajo fueron reportados por Hansen et al. (2002), al utilizar hemacitómetros y espectrofotometría en porcino con valores de 10,2 y $6,3 \%$, respectivamente. 
Los resultados del presente estudio revelan que los verracos Duroc tuvieron mayores concentraciones de espermatozoides que los Pietrain, para todos los métodos de estimación. Esto coincide con los trabajos de Banaszewska et al. (2009), Kondracki et al. (2011), Wolf y Smital (2009) y Smital et al. (2004) en verracos Pietrain y Duroc, respectivamente. Además, se ha descrito una relación entre los parámetros morfométricos de los espermatozoides y la concentración espermática en los eyaculados. Los espermatozoides en los eyaculados menos concentrados tienden a presentar cabezas más grandes que los espermatozoides en los eyaculados con concentraciones más altas. Esto es confirmado en estudios con verracos Pietrain (Banaszewska et al. 2009), Duroc (Kondracki et al. 2011) y en bovino (Kondracki et al. 2012), que han demostrado que los espermatozoides tienen cabezas más grandes en los eyaculados en que la concentración de esperma es más baja.

No hubo diferencias entre los métodos de medición de la concentración cuando el intervalo entre extracciones seminales fue mayor de 9 días. Cuando el intervalo fue menor de 9 días, sólo hubo diferencias entre EF y CASA. Dentro del método no hubo diferencias en intervalos entre extracciones superiores e inferiores a 9 días, lo que indica que el efecto del descanso a los verracos, entre extracciones seminales, no mejora significativamente la concentración en el eyaculado. La interacción entre la concentración espermática y el intervalo entre extracciones seminales fue significativa para el método CASA y el nivel de asíntota fue determinado en 7,5 días, lo que indica que intervalos superiores, a una semana de descanso entre extracciones seminales, no mejoraría significativamente la concentración de espermatozoides en el eyaculado. De acuerdo con los trabajos de Rutten et al. (2000), Frangež et al. (2005) y Smital (2009) si se considera el nivel asintótico estimado en el presente trabajo, se podría proponer un intervalo entre extracciones entre 7 a 10 días como apropiado para optimizar la calidad seminal desde el punto de vista biológico. Rutten et al. (2000) investigaron intervalos de recolección de 1 a 10 días y encontraron que el mayor número de dosis por recolección se puede generar para intervalos de 7 a 10 días.

En todos los métodos de estimación, la concentración fue menor cuando el volumen de eyaculado fue mayor, esto podría explicarse por un efecto de dilución que hace que por unidad de volumen haya menos espermatozoides, porque hay más plasma seminal. Se ha descrito en trabajos previos (Wolf y Smital 2009), que el número total de espermatozoides es función del volumen y la concentración espermática. Sin embargo, cuando los volúmenes de eyaculado son bajos, los métodos estiman con mayor variabilidad la concentración espermática al encontrarse una diferencia de medias significativa entre el EF y el sistema CASA de 83,09x $10^{6} \cdot \mathrm{ml}^{-1}$.

Al analizar el efecto de época sobre la concentración seminal, se encontró que durante la estación seca la concentración espermática fue mayor que en la lluviosa, independientemente del método utilizado para estimación. Dentro de época solo hubo diferencias en la estimación de la concentración entre los métodos EF y CASA en la estación lluviosa. En condiciones tropicales, la reproducción de los animales es continua, sin embargo, el efecto de la temperatura, humedad relativa y precipitaciones pueden afectar la eficiencia reproductiva (Weitze 2000, RodríguezMartínez y Wallgren 2000).

El coeficiente de correlación proporciona una estimación de la asociación lineal entre las mediciones obtenidas por los métodos de estimación de la concentración. Mide la fuerza de asociación y, en la literatura se ha utilizado en muchos estudios, como comparación entre diferentes procedimientos para determinar la concentración del esperma (Prathalingam et al. 2006). Las técnicas de medición de la concentración espermática se han basado en diseños estadísticos correlativos para determinar la relación entre los de estimación y factores como exactitud, precisión y repetibilidad (Rigby et al. 2001, Evenson et al. 1993).

Hubo una interacción significativa entre el método de estimación de la concentración 
espermática y el intervalo entre extracciones seminales. Los métodos de medición tienden a presentar más variabilidad cuando los verracos son sometidos a frecuencias mayores de extracción de semen y el efecto se ve más pronunciado en el sistema CASA, seguido del espermadensímetro y por último, el espectrofotómetro. Una mayor frecuencia de extracciones podría provocar que haya más células inmaduras, epiteliales y residuos celulares, que fuesen reconocidos como espermatozoides en el análisis de imagen y, como resultado de ello, la concentración es mayor (Eustache et al. 2001). El efecto de la interacción también sugiere que mantener los verracos en descanso entre extracciones de semen, no tiende a mejorar la concentración espermática y para el sistema CASA se estableció un nivel asintótico de 7,5 días, por encima del cual, la concentración espermática no se incrementa significativamente. Otros autores recomiendan una frecuencia de recolección de una vez por semana (Rozeboom 2000), mientras que en otros trabajos se ha establecido que para optimizar el uso de los verracos se realicen colecciones 3 veces cada 15 días (Flowers 2002). Langendijk (2001), Soede et al. (2002), y Steverink (1999) recomiendan usar los verracos cada 6 días, lo que es muy similar a lo encontrado en este trabajo a partir de 7,5 días como punto asintótico de intervalo entre extracciones.

La interacción entre método de estimación de concentración y grupo racial fue significativa. Se encontró que en la raza Duroc existe una mayor exactitud al medir la concentración que en Pietrain, donde la mayor variabilidad fue presentada en la evaluación con el sistema CASA. Se ha reportado que hay variación individual (Kondracki et al. 2011) y entre raza (Saravia et al. 2007), lo que puede explicar los resultados observados en el presente trabajo. La menor concentración observada en los verracos Pietrain, al utilizar el método CASA, puede deberse a las condiciones particulares del análisis de imagen, como por ejemplo el reconocimiento de espermatozoides estáticos, por lo que es recomendable después de cada análisis de semen con equipos automatizados, que los técnicos de laboratorio realicen una revisión de los campos capturados para evitar la introducción de sesgos en los resultados.

Debido a que una gráfica de superficie solo muestra 2 variables categóricas por vez, el resto de las variables se mantuvieron a un nivel constante. La edad se mantuvo fija en 36 meses. En el presente trabajo, los valores más altos de concentración coincidieron con los menores de volumen de eyaculado y mayores intervalos entre extracciones y viceversa. Bonet et al. (1991) reportaron mayores concentraciones de semen cuando el intervalo entre extracciones fue semanal. Por otro lado, Wolf y Smital (2009) han reportado correlaciones entre volumen de eyaculado y concentración espermática del orden de $-0,61$, lo que refuerza los resultados observados en este trabajo. La relación entre volumen e intervalo entre extracciones seminales sobre la concentración de espermatozoides, también ha sido analizada por Frangež et al. (2005), quienes observaron que la concentración se disminuyó cuando se incrementó el volumen eyaculado y se aumentó la frecuencia de extracciones de semen en los verracos. Aunque un intervalo entre extracciones mayor puede mejorar la calidad seminal, Rutten et al. (2000) demostraron que desde el punto de vista económico y de producción de dosis seminales, intervalos menores mejoran la utilidad de la granja, sin embargo, esto puede conllevar a una disminución de la calidad y valor biológico de los espermatozoides (Strzezek et al. 1995) similar a la apoptosis de las células somáticas.

El análisis de regresión no lineal, entre edad de los verracos y concentración, mostró una reducción del mismo conforme se incrementó la edad y para todos los métodos de estimación. Esto concuerda con algunos trabajos previos (Flowers 2002), donde se recomienda mantener a los verracos hasta los 24 meses, ya que su calidad seminal disminuye. Aunque la tendencia fue la misma en todos los métodos de estimación, la disminución de la concentración fue más evidente en los métodos ED y EF que en el sistema CASA. La elevada concentración mostrada en los verracos jóvenes se corresponde con lo reportado por Clark et al. (2003) en verracos desde 8 hasta 
14 meses de edad, con pocos cambios a partir de entonces y tendiendo a disminuir con la edad (Rutten et al. 2000), lo que es coincidente con el presente trabajo.

En conclusión, los métodos de estimación de la concentración espermática presentan una variabilidad en los resultados que debe ser minimizada, mediante la identificación de fuentes de variación para mejorar la exactitud y asegurar un número adecuado de dosis seminales. El intervalo entre extracciones seminales óptimo se encuentra entre 7 y 10 días, con un nivel asintótico sobre 7,5 días. La variación inter-razas demostró que los verracos Duroc presentan eyaculados más concentrados que los Pietrain, independientemente del método de estimación de la concentración, sin embargo, en la raza Pietrain se presentó mayor variabilidad de resultados entre los métodos. El volumen eyaculado presentó una relación negativa con la concentración espermática en todos los equipos de medición. En época seca, los animales presentan valores de concentración espermática mayores que en época lluviosa. La concentración de semen disminuye conforme se incrementa el volumen de eyaculado y la edad de los verracos en los 3 métodos de estimación, pero en el caso de la edad, el sistema CASA presenta una pendiente menor que en los métodos ED y EF.

\section{AGRADECIMIENTOS}

Los autores agradecen al Programa de Producción Agropecuaria (PPA) de la Escuela de Agronomía del Instituto Tecnológico de Costa Rica y a los funcionarios Sr. Juan Orozco y DPA. Rodrigo Solís por el apoyo recibido durante el desarrollo de la investigación.

\section{LITERATURA CITADA}

Amann, R; Waberski, D. 2014. Computer-assisted sperm analysis (CASA): Capabilities and potential developments (en línea). Theriogenology 81(1):5-17.e3. DOI: https://doi.org/10.1016/J. THERIOGENOLOGY.2013.09.004

Banaszewska, D; Kondracki, S; Wysokińska, A. 2009. Effect of sperm concentration on ejaculate for morphometric traits of spermatozoas of the pietrain breed boars. J Cent Eur Agr 10:383-396.

Bonet, S; Briz, M; Fradera, A. 1991. The sperm quality and fertility of boars after two different ejaculation frequencies (en línea). Article Scientia gerundensis 17:77-84. Consultado 6 Jun. 2018. Disponible en https://www.raco.cat/index.php/Scientia/article/ viewFile/45463/54997

Bonet, S; Casas, I; Holt, WV; Yeste, M. 2013. Boar reproduction: fundamentals and new biotechnological trends. s.l. Berlin, Alemania, Springer-Verlag. p. 632.

Bortolozzo, FP; Wentz, I; Dallanora, D. 2005. Situação atual da inseminação artificial em suínos (en línea). Acta scientiae veterinariae 33(1):17-32. Consultado 3 Jun. 2018. Disponible en http://www.lume.ufrgs.br/ handle/10183/20025

Brito, LFC; Althouse, GC; Aurich, C; Chenoweth, PJ; Eilts, BE; Love, CC; Luvoni, GC; Mitchell, JR; Peter, AT; Pugh, DG; Waberski, D. 2016. Andrology laboratory review: Evaluation of sperm concentration (en línea). Theriogenology 85(9):1507-1527. DOI: https://doi. org/10.1016/j.theriogenology.2016.01.002

Ciereszko, A; Ottobre, JS; Glogowski, J. 2000. Effects of season and breed on sperm acrosin activity and semen quality of boars. (en línea). Animal reproduction science 64(1-2):89-96. Consultado 3 Jun. 2018. Disponible en http://www.ncbi.nlm.nih. gov/pubmed/11078969

Clark, SG; Schaeffer, DJ; Althouse., GC. 2003. B-mode ultrasonographic evaluation of paired testicular diameter of mature boars in relation to average total sperm numbers. Theriogenology 60:1011-1023.

Davis, R; Gravance, C; Casey, P. 1993. Automated morphometric analysis of stallion spermatozoa. Am J Vet Res 54(11):1808-1811.

Ehlers, J; Behr, M; Bollwein, H; Beyerbach, M; Waberski, D. 2011. Standardization of computer-assisted semen analysis using an e-learning application (en línea). Theriogenology 76(3):448-454. DOI: https://doi. org/10.1016/j.theriogenology.2011.02.021

Eljarah, A; Chandler, J; Jenkins, J; Chenevert, J; Alcanal, A 2013. Usefulness of hemocytometer as a counting chamber in a computer-assisted sperm analyzer (CASA). Anim. Reprod 10(4):708-711.

Emik, LO; Sidwell, GM. 1947. Factors Affecting the Estimation of Concentration of Sperm in Ram's Semen by the Photoelectrometric Method (en línea). Journal of Animal Science 6(4):467-475. DOI: https://doi.org/10.2527/jas1947.64467x

Eustache, F; Jouannet, P; Auger, J. 2001. Evaluation of Flow Cytometric Methods of Measure Human Sperm Concentration. Journal of Andrology 22(4):558-567.

Evenson, D; Parks, J; Kaproth, M; Jost, L. 1993. Rapid determination on sperm cell concentration in bovine semen by flow cytometry. Journal of Dairy Science 76:86-94. 
Flowers, WL. 1997. Management of boars for efficient semen production. (en línea). Journal of Reproduction and Fertility. Supplement 52:67-78. Consultado 3 Jun. 2018. Disponible en http://www.ncbi.nlm.nih.gov/ pubmed/9602720

Flowers, WL. 2002. Using reproductive biology to improve suboptimal reproductive performance. In Proccedings of the 17th IPVS Congress. Ames Iowa, USA, s.e. p. 43-48.

Frangež, R; Gider, T; Kosec, M. 2005. Frequency of boar ejaculate collection and its influence on semen quality, pregnancy rate and litter size. Acta Vet. (Brno) 74:265-273.

Graffer, T; Solbu, H; Filseth, O. 1988. Semen production in artificial insemination bulls in Norway (en línea). Theriogenology 30(5):1011-1021. DOI: https://doi. org/10.1016/S0093-691X(88)80064-5

Hancock, J; Hovell, G. 1959. The collection of boar semen. The Veterinary Record 71:664-665.

Hansen, C; Christensen, P; Stryhn, H; Hedeboe, A; Rode, M; Boe-Hansen, G. 2002. Validation of the FACSCount AF System for Determination of Sperm Concentration in Boar Semen (en línea). Reproduction in Domestic Animals 37(6):330-334. DOI: https://doi.org/10.1046/ j.1439-0531.2002.00367.x

Holt, C; Holt, WV; Moore, H; Reed, H; Curnock, R. 1997. Objectively measured boar sperm motility parameters correlate with the outcomes of on-farm inseminations: results of two fertility trials. Journal of Andrology 18:312-323.

Keel, B. 2002. Quality control, quality assurance, and proficiency testing in the andrology laboratory. Arch Androl 48:417-431.

Kondracki, S; Banaszewska, D; Mielnicka, C. 2005. The effect of age on the morphometric sperm traits of domestic pig (Sus scrofa domestica). Cell Mol Biol Lett 10(1):3-13.

Kondracki, S; Banaszewska, D; Wysokińska, A; Iwanina, M. 2012. The effect of sperm concentration in the ejaculate on morphological traits of bull spermatozoa. Folia Biol- Krakow 60 (1-2):85-91.

Kondracki, S; Wysokińska, A; Iwanina, M; Banaszewska, D; Sitarz, D. 2011. Effect of sperm concentration in an ejaculate on morphometric traits of spermatozoa in Duroc boars (en línea). Polish Journal of Veterinary Sciences 14(1):35-40. DOI: https://doi.org/10.2478/ v10181-011-0005-z

Krueger, C; Rathl, D; Johnson, LA. 1999. Low dose insemination in synchronized gilts (en línea). Theriogenology 52:1363-1373. Consultado 3 Jun. 2018. Disponible en https://pubag.nal.usda.gov/ download/34154/PDF

Langendijk, P. 2001. Is there a future for the boar? The role of boar stimuli in reproductive processes around estrus in the pig. s.l., Wageningen University, The Netherlands.
Matson, P. 1995. External quality assessment for semen analysis and sperm antibody detection: results of a pilot scheme. Human Reproduction 10:620-625.

Mortimer, D; Aitken, R; Mortimer, S; Pacey, A. 1995. Workshop report: clinical CASA-the ques for consensus. Reproduction, Fertility and Development 7:951-959.

Mortimer, S. 2000. CASA-Practical Aspects (en línea) Journal of Andrology 21(4):515-524. DOI: https:// doi.org/10.1002/J.1939-4640.2000.TB02116.X

Murgas, L; Lima, D; Alvarenga, A; Zangeronimo, M; Oberlender, G; Oliveira, S. 2010. Estudo comparativo de diferentes técnicas de avaliação da concentração espermática em suínos comparative study of different techniques of evaluation of spermatic concentration in swines (en línea). Arch. Zootec 59(227):463-466. Consultado 6 Jun. 2018. Disponible en http://scielo. isciii.es/pdf/azoo/v59n227/art17.pdf

Neuwinger, J; Behre, H; Nieschlag, E. 1990. External quality control in the andrology laboratory: an experimental multicenter trial. Fertil Steril 54:308-314.

Prathalingam, NS; Holt, WW; Revell, SG; Jones, S; Watson, PF. 2006. The Precision and Accuracy of Six Different Methods to Determine Sperm Concentration (en línea). Journal of Andrology 27(2):257-262. DOI: https://doi.org/10.2164/jandrol.05112

Rigby, S; Varner, D; Thompson, J; Love, C; Brinsko, S; Blanchard, T. 2001. Measurement of sperm concentration in stallion ejaculates using photometric or direct sperm enumeration techniques. Proc Ann Conv Am Assoc Equine Pract 47:236-238.

Rijsselaere, T; Van Soom, A; Maes, D; Kruif, A de. 2004 Effect of technical settings on canine semen motility parameters measured by the Hamilton-Thorne analyzer. Theriogenology 60(8):1553-1568. DOI: https://doi.org/10.1016/S0093-691X(03)00171-7

Rodríguez-Martínez, H; Wallgren, M. 2000. Factores que influencias la calidad espermática en verracos en inseminaçao artificial em suínos. In III Simposio Internacional MINITUB. Flores da Cunha, RS, Brasil, s.e. p. 34-41.

Rozeboom, K. 2000. Guidelines for Boar Semen Processing: Initial Analysis and Sort. In Proccedings of the 2000 North Carolina Pork Conference. Service, NCSUCE (ed.). North Carolina State, USA, s.e.

Rutten, SC; Morrison, RB; Reicks, D. 2000. Boar stud production analysis. Swine Health Prod 8:11-14.

Saravia, F; Núñez-Martínez, I; Morán, J; Soler, C; Muriel, A; Rodríguez-Martínez, H; Peña, F. 2007. Differences in boar sperm head shape and dimensions recorded by computer-assisted sperm morphometry are not related to chromatin integrity (en línea). Theriogenology 68(2):196-203. DOI: https://doi org/10.1016/j.theriogenology.2007.04.052

Smital, J. 2009. Effects influencing boar semen (en línea). Animal reproduction science 110(3-4):335-46. DOI: https://doi.org/10.1016/j.anireprosci.2008.01.024 
Smital, J; Sousa, LL De; Mohsen, A. 2004. Differences among breeds and manifestation of heterosis in AI boar sperm output. Animal Reproduction Science 80:121-130.

Soede, N; Langendijk, P; Kemp, B. 2002. Optimum strategies in artificial insemination, with emphasis on the timing relative to ovulation and the role of the boar. In Proceedings of the 17th IPVS Congress. s.1., s.e. p. 15-23.

Steverink, DWB. 1999. Optimizing insemination strategies in pigs. s.l., Wageningen University. The Netherlands.

Strzezek, J; Kordan, W; Glogowski, J; Wysocki, P; Borkowski, K. 1995. Influence of semen-collection frequency on sperm quality in boars, with special reference to biochemical markers. Reproduction in Domestic Animals 30:85-94.

Tomlinson, MJ; Pooley, K; Simpson, T; Newton, T; Hopkisson, J; Jayaprakasan, K; Jayaprakasan, R; Naeem, A; Pridmore, T. 2010. Validation of a novel computer-assisted sperm analysis (CASA) system using multitarget-tracking algorithms (en línea). Fertility and Sterility 93(6):1911-1920. DOI: https:// doi.org/10.1016/j.fertnstert.2008.12.064

Valverde, A; Madrigal-Valverde, M. 2018. Sistemas de análisis computadorizado de semen en la reproducción animal (en línea). Agronomía Mesoamericana DOI: https://doi.org/10.15517/ma.v29i2.30613

Valverde, A; Madrigal-Valverde, M; Caldeira, C; Bompart, D; Núñez de Murga, J; Arnau, S; Soler, C. 2018. Effect of frame rate capture frequency on sperm kinematic parameters and subpopulation structure definition in boars, analyzed with a CASA-Mot system (en línea). Reproduction in Domestic Animals <in press>. DOI: https://doi.org/10.1111/rda.13320

Valverde, A; Madrigal-Valverde, M; Camacho-Calvo, M; Zambrana-Jiménez, A; López, L. 2018. Efecto de la composición racial sobre la calidad espermática de verracos (en línea). Agronomía Mesoamericana 29(3):485-506. DOI: https://doi.org/10.15517/ ma.v29i3.32445

Vásquez, F; Soler, C; Camps, P; Valverde, A; GarcíaMolina, A. 2016. Spermiogram and sperm head morphometry assessed by multivariate cluster analysis results during adolescence (12-18 years) and the effect of varicocele. Asian Journal of Andrology 18(6):824-830. DOI: https://doi. org/10.4103/1008-682X.186873

Vianna, W; Gonçalves, B; Namindome, A; Campos, A; Henrique, P; Mazza, PH; Pinese, ME; de Sant Anna, A De; Moretti, A. 2004. Estudo Comparativo da Eficiência de Diferentes Técnicas de Mensuração da Concentração Espermática em Suínos Efficiency of Different Measurement Techniques of Sperm Concentration in Swine. R. Bras. Zootec. 33(6):2054-2059.

Weitze, K. 2000. Infertilidade estacional no suino. In III Simposio Internacional "Inseminaçào Artificial em Suinos." Universidade Federal do Rio Grande do Sul, Brasil, s.e. p. 50-55.

Wolf, J; Smital, J. 2009. Quantification of factors affecting semen traits in artificial insemination boars from animal model analyses1 (en línea). Journal of Animal Science 87(5):1620-1627. DOI: https://doi. org/10.2527/jas.2008-1373

World Health Organization. 2010. WHO laboratory manual for the examination and processing of human semen. s.l., World Health Organization. Geneva, Switzerland. $271 \mathrm{p}$.

Zrimšek, P. 2011. Evaluation of a new method and diagnostic test in semen analysis. In Artificial insemination in farm animals. In Manafi ME (ed.). Rijeka, Croatia, InTech. p. 131-152.

Todos los derechos reservados. Universidad de Costa Rica. Este artículo se encuentra licenciado con Creative Commons Reconocimiento-NoComercial-SinObraDerivada 3.0 Costa Rica. Para mayor información escribir a rac.cia@ucr.ac.cr 
